\section{$\underset{\substack{\text { hommes } \\ \text { \& migrations }}}{ }$}

\section{Hommes \& migrations}

Revue française de référence sur les dynamiques

migratoires

1307 | 2014

L'Afrique qualifiée dans la mondialisation

\title{
Arbi, bled, cagna, y a bon..., l'altérité langagière dans la Grande Guerre
}

\section{Odile Roynette}

\section{QpenEdition \\ Journals}

Édition électronique

URL : http://journals.openedition.org/hommesmigrations/2922

DOI : 10.4000/hommesmigrations.2922

ISSN : 2262-3353

Éditeur

Musée national de l'histoire de l'immigration

Édition imprimée

Date de publication : 1 juillet 2014

Pagination : 184-189

ISBN : 978-2-919040-28-5

ISSN : $1142-852 X$

\section{Référence électronique}

Odile Roynette, « Arbi, bled, cagna, y a bon..., l'altérité langagière dans la Grande Guerre », Hommes \& migrations [En ligne], 1307 | 2014, mis en ligne le 15 janvier 2015, consulté le 22 septembre 2020. URL : http://journals.openedition.org/hommesmigrations/2922 ; DOI : https://doi.org/10.4000/ hommesmigrations.2922 


\title{
CHRONIQUE DE GUERRE
}

\section{$A R B I, B L E D, C A G N A$, YABON...}

\section{L'ALTÉRITÉ LANGAGIÈRE DANS LA GRANDE GUERRE}

\author{
ODILE ROYNETTE, maître de conférences en histoire contemporaine, \\ université de Franche-Comté et Centre d'histoire de Sciences Po
}

"Non! L'argot de la guerre n'est pas un phénomène extraordinaire ni une langue créée de toutes pièces. C'est autre chose et c'est beaucoup mieux : c'est la transformation de l'argot de caserne, profondément modifié par la vie guerrière, enrichi par les apports de l'argot parisien, des provincialismes de bonne frappe et des mots exotiques, que nos troupes ont empruntés aux contingents coloniaux et étrangers, ou aux populations indigènes avec lesquelles elles ont été en contact dans des expéditions lointaines'." Par ce jugement, l'éminent linguiste français, Albert Dauzat, auteur d'une enquête publiée au printemps 1918 sur l'argot de la guerre, mettait en évidence l'apport colonial à la langue française. Il lui conférait une visibilité nouvelle dans le contexte d'un conflit mondialisé qui affecta durement les populations dites "indigènes".

La mobilisation des contingents coloniaux, rappelons-le, concerna entre 550 ooo et 600 ooo hommes au minimum, engagés volontaires ou enrôlés de force par le biais de la conscription imposée aux contingents créoles, aux Sénégalais des quatre communes (Saint-Louis, Rufisque, Dakar et Gorée) puis aux Algériens à partir de l'automne 1915 et enfin aux Tunisiens. Sur ce nombre, 450 ooo furent dépêchés en Europe comme travailleurs cantonnés à l'arrière et comme combattants composés en majorité de Maghrébins (190 0oo, principalement des Algériens) et de tirailleurs sénégalais (134 000, originaires à $45 \%$ du Haut-Sénégal et du Niger, $19 \%$ de la Guinée, 14 \% de la Côte d'Ivoire et $7 \%$ du Dahomey), les autres provenant de toutes les parties de l'empire ${ }^{2}$. Ces hommes ont payé un lourd tribut à l'effort de guerre européen avec des pertes qui semblent comparables à celles des unités métropolitaines 3 . En outre, la puissance de destruction de la Grande Guerre a touché plus particulièrement le continent africain, devenu le lieu d'une concurrence pour la prédation des territoires coloniaux détenus par l'Allemagne, notamment ceux, faiblement défendus, de l'Afrique occidentale, Cameroun et Togo. De sorte que l'ensemble du monde colonial fut alors, bien malgré lui, projeté en pleine lumière.

Cette présence coloniale dans la Grande Guerre a eu des effets linguistiques auxquels nous allons nous intéresser ici. Avant 1914, l'intérêt pour les néologismes créés par les troupes au contact de la réalité coloniale comme la prise en compte des emprunts aux langues des colonisés étaient restés confinés à la sphère des argotiers, érudits soucieux de détecter les inventions langagières et animés par un fort goût du pittoresque. Avec la Première Guerre mondiale, l'attention accordée à 
ces créations se modifia, de même que s'accentua l'intérêt des lettrés pour ces créations "exotiques", pour reprendre l'adjectif utilisé par Albert Dauzat. Quel sens, toutefois, accorder à cette curiosité accrue? Manifeste-t-elle une modification du rapport à l'autre porteur d'une acculturation réciproque ou témoigne-t-elle plutôt d'un enracinement du sentiment de la différence avec les peuples colonisés?

\section{L'empreinte du XIX ${ }^{\mathrm{e}}$ siècle colonial}

La Première Guerre mondiale ne constitua pas une rupture dans l'histoire du regard posé sur un vocabulaire issu de l'expérience coloniale. Dès la seconde moitié du XIXe siècle, les premiers dictionnaires consacrés au français populaire par des auteurs fascinés dans une perspective romantique, par le peuple et les bas-fonds avaient accordé une place à l'argot des soldats alors issus des milieux sociaux les plus modestes ${ }^{4}$. Retenons principalement celui de Lorédan Larchey, Les Excentricités de la langue française, en 1860, ou celui d'Alfred Delvau, Dictionnaire de la langue verte, publié quelques années plus tard, en 1866. Ces deux lexicographes, étrangers à la linguistique savante, observèrent dans l'armée un creuset d'épanouissement et de diffusion de la langue orale populaire. Le service militaire, très inégalitaire jusqu'en 1872, retenait alors en majorité des paysans et des ouvriers qui servaient pendant de longues années et qui furent les artisans de la conquête coloniale. En outre, les territoires colonisés par l'armée française, principalement l'Algérie et la Tunisie, furent choisis comme lieux de relégation privilégiés des détenus militaires, composés de condamnés à de lourdes peines par les conseils de guerre métropolitains pour des délits ou des crimes. S'y ajoutaient des soldats considérés en métropole comme "incorri- gibles" et rassemblés dans les compagnies de discipline, les "biribi" de sinistre mémoire. Ces unités formèrent un laboratoire de création et de diffusion d'un français populaire employé par une population de délinquants civils usagers de l'argot des bagnes et des prisons. II était mélangé à un argot militaire marqué par des mots créés dans le contexte colonial ou empruntés à l'arabe, à l'algérien notamment, comme le fit remarquer dès 1971 l'historien du fait colonial Claude Liauzu5. Dès le début des années 1860 et jusqu'à la Belle Époque, les dictionnaires d'argot recueillirent cette manne lexicale restée en marge du français académique. Il est possible d'y distinguer deux catégories de mots.

\section{La domination des emprunts à l'arabe algérien ou au berbère}

La première catégorie, formée à partir du français soutenu, regroupe des mots bâtis sur des déformations morphologiques ou sur des métaphores. Le premier sous-ensemble - moins d'une dizaine de mots avant 1870 - se concentre sur la désignation de soi et de l'autre, les mots servant ici visiblement à apprivoiser une étrangeté constitutive de la relation coloniale. Arbi, et ses dérivés Arbicot et Bicot, formés à partir du mot "Arabe", sont parmi les plus anciens, comme le Turco, déformation argotique du mot "tirailleur", employé pour nommer les tirailleurs indigènes de l'armée d'Afrique qui concentrait avant le début du XXe siècle le gros des troupes indigènes de l'Afrique du Nord. Quant aux métaphores, le zéphir désigna très tôt le soldat des compagnies de discipline, par analogie avec le vent d'ouest dont la légèreté pouvait se comparer à la mobilité de ces combattants employés sur le champ de bataille comme tirailleurs. Un peu plus tard, apparut le mot cafard, par allusion au gros insecte noir qui se complaît dans l'obscurité. II 


\section{CHRONIQUE DE GUERRE}

fut répandu au sein de l'armée d'Afrique, notamment parmi les hommes de la Légion étrangère et des bataillons d'infanterie légère, pour désigner la détresse morale de ces soldats européens brutalement déracinés et enclins au mal du pays. La nostalgie contribua, dans des proportions difficiles à établir, à la surmortalité des troupes déployées dans les colonies et fut l'objet de l'attention des médecins militaires et du commandement soucieux, à l'image du général de Castellane dans les années 1840, par exemple, de doter les postes algériens d'un réseau stable de lieux de divertissement (théâtres, cafés et bibliothèques) capables d'enrayer "les idées noires, si souvent en Afrique avant-coureurs de Dès les années 1860, la nostalgie et de la mort ${ }^{6 "}$. les argotiers enregistrèrent La Grande Guerre allait donner une autre ampleur à ce mal et transformer le sens du terme.

Un second ensemble fut formé par les emprunts à la langue des colonisés et constitua de loin le plus important en nombre. Dès les années 1860, les argotiers enregistrèrent l'apparition dans l'usage courant de mots comme beseff (ou bezef), synonyme de "beaucoup", chouia (peu), guitoune (tente de campement), kif-kif (ou kifkif bourricot) équivalent à "le même" ou "pareillement", gourbi (abri précaire, hutte), kébir (chef), macache (non, pas du tout), razzia (expédition punitive), auxquels vint s'ajouter entre les années 1880 et 1914 une deuxième strate de termes devenus usuels comme bled (le village), cagna (abri fragile), caoua (café), clebs (chien), nouba (fête), ramdam (bruit), sidi (soldat indigène), toubib (médecin-major) ou, plus rarement cité, bougnoul(e) pour un soldat noir. II ne saurait être question de restituer ici l'étymologie de chacun de ces mots7, mais plutôt de souligner quelques caractères majeurs de ces apports lexicaux.

Premier constat, celui de la domination dans ce lexique des mots empruntés à l'arabe algérien ou au berbère, signe de la place prépondérante acquise avant 1914 par l'Algérie, et plus tardivement par la Tunisie, dans l'entreprise de domination française comme dans l'imaginaire colonial. Les territoires de l'Afrique noire plus tardivement conquis, et qui ne furent pas des colonies de peuplement, restèrent à la marge de ces transferts culturels. De nombreux lexicographes avant 1914 relevèrent la place des unités stationnées en Afrique du Nord, numériquement les plus nombreuses il est vrai, dans ces métissages linguistiques et leur transfert vers les usages oraux populaires au retour des colonies grâce surtout aux citadins, notamment aux soldats parisiens, qui diffusèrent ce "sabir" dans l'argot général.

La dimension méprisante, voire ouvertement raciste de ce vocabulaire, si frappante de nos jours, doit être attentivement questionnée. Les dictionnaires d'argot publiés avant 1914 restèrent extrêmement prudents sur la stigmatisation de l'autre contenue dans le dévoilement de ce vocabulaire. Les notices s'efforcèrent de naturaliser ces mots sans rien dire des jugements dévalorisants qu'ils pourraient contenir, ce qui ne signifie pas qu'ils étaient absents, bien au contraire. Ainsi l'historien Gilles Manceron a relevé la place au café-concert de chansons en "petit nègre" qui ont contribué à banaliser la hiérarchie des races à l'extrémité de laquelle étaient assignées les populations noires. De même, en 1897, il a souligné le succès de la 
chanson Kif-kif bourrico qui tournait en dérision le docteur Philippe Grenier, député de Pontarlier dans le Jura, qui, converti à l'islam, s'était présenté à la Chambre vêtu d'un burnous blanc ${ }^{8}$.

\section{La diffusion de l'apport colonial pendant la Grande Guerre}

Le premier conflit mondial se caractérisa à la fois par une diffusion de ce patrimoine colonial dans les usages communs et par une présence encore accrue de l'élément africain, deux évolutions qui n'échappèrent pas à l'attention des linguistes. En 1915, l'érudit d'origine roumaine Lazare Sainéan, auteur d'un des premiers lexiques de l'argot des tranchées, soulignait déjà ce double mouvement: "Depuis la conquête de l'Algérie et surtout depuis l'institution des troupes africaines, nombre de termes algériens, appartenant soit à l'arabe du Nord, soit au mélange linguistique connu sous le nom de sabir, ont pénétré jusqu'à Paris et ont trouvé accès dans le bas langage de la capitale. D'autres, restés confinés dans les sphères militaires, se tenaient à l'écart du courant général. Depuis cette guerre qui a réuni dans la même tranchée les poilus de France et ceux de son empire colonial, ces termes spéciaux ou rarement employés sont en train de se répandre et de se généraliser $9 . "$

À l'appui de sa démonstration empreinte d'esprit cocardier, il citait une série de mots fort répandus parmi les soldats: barda ou bardin le sac et l'équipement du soldat), guitoune, gourbi, kasba (la maison), nouba, toubib et cagna(t). Très critique à l'égard de la documentation comme de la méthode utilisées par Sainéan, le linguiste combattant Marcel Cohen ${ }^{10}$, dont les pre- miers travaux avant guerre témoignaient de son intérêt pour les argots et de sa sensibilité à la question des métissages linguistiques - il avait publié en 1912 Le Parler arabe des Juifs d'Alger-, reconnaissait en 1916 au moins un mérite à Sainéan : celui d'avoir "bien discerné l'originalité de l'apport colonia/"1".

Les études ultérieures Le premier conflit mondial se caractérisa à la fois par une diffusion de ce patrimoine colonial dans les usages publiées durant le conflit ou à son issue immédiate, notamment les deux plus sérieuses, celle d'Albert Daucommuns et par une présence encore accrue de l'élément africain, deux évolutions zatetcelle de Gaston Esnault qui n'échappèrent pas (Le Poilu tel qu'il se parle, à l'attention des linguistes. 1919), confirmèrent la pertinence de ce constat tout en en précisant davantage la nature. Albert Dauzat, qui avait mené à partir de 1917 une enquête à distance en recueillant par écrit les réponses de quelque 200 mobilisés relatives au vocabulaire des combattants, accorda une place non négligeable aux mots forgés dans les corps étrangers et coloniaux et à leur vulgarisation dans le français populaire. Plusieurs de ses correspondants, soldats dans des unités stationnées outre-mer avant 1914, en témoignèrent, comme ce brigadier vétérinaire au 8 régiment de marche de chasseurs d'Afrique, Ducousso, qui écrivait à Dauzat depuis le front d'Orient une liste de mots et leur définition, sur laquelle figuraient : "indigène en général : bicot", "tirailleur annamite : gnacoué", "tirailleur algérien : couss-couss, arbi", "sous-officier : chaouch"12", ou encore ce sous-officier à la Légion étrangère, Louis Mise, lui aussi affecté à l'armée d'Orient, attestant l'usage de "thiou-thiou : riz en langue annamite" ou de guitoune pour "abri'13". 


\section{CHRONIQUE DE GUERRE}

\section{Argot des Parisiens, argot des tirailleurs}

D'autres témoignages restituent la profondeur historique de ce vocabulaire, comme celui de ce soldat lettré, Antonin Duraffour, professeur d'allemand au lycée de Grenoble à la mobilisation et féru de philologie. Devenu officier interprète dans l'état-major de son corps d'armée, il adresse plusieurs missives à Dauzat, notamment celle du 21 avril 1917 dans laquelle il détaille l'usage de certains mots d'origine coloniale. Ainsi de toubib qu'il a "entendu pour la première fois à la caserne en 1905 au mess des sous-

L'intégration de

ce vocabulaire d'origine coloniale, dont les linguistes ont montré la dissémination

dans le français oral populaire, s'est révélée profondément ambiguë. officiers que j'ai fréquenté avec un adjudant et un médecin auxiliaire. L'adjudant n'était pas africain. Le mot était d'un emploi peut-être restreint à cette époque: je l'ai entendu très souvent dans mon $E M$ [état-major] dès le début des hostilités'14". En mai 1917, un certain G. Mayer, trésorier aux armées, signale à Dauzat : "Toubib et caoua étaient déjà d'un usage courant dans certaines unités au XXe corps bien avant la guerre (en 1908-1909, aux $8^{e}$ et $39^{e}$ d'artillerie). Certains disaient caouar au lieu de caoua'5." Avant 1914, le rôle joué par les soldats parisiens, nombreux à être affectés dans les unités stationnées à l'est de la France (le XXe corps d'armée était lorrain avant guerre), dans l'incorporation de ce lexique à l'argot général et dans sa dissémination, était ainsi pleinement souligné Enfin, certains observateurs enregistrèrent pen- dant le conflit l'importation puis la diffusion, restée semble-t-il localisée, d'un lexique propre aux tirailleurs indigènes pendant la seconde moitié de la guerre. Le phénomène se répandit alors dans le Midi de la France, plus précisément dans la Provence côtière, où furent concentrées à partir de l'hiver 1915-1916 les principales structures pour héberger ces combattants pendant les mois les plus froids, à défaut de leur octroyer la possibilité de rentrer au pays pendant leur permission. Ce droit ne leur fut concédé qu'en décembre 1917, mais son application resta limitée par les offensives de l'année $1918^{16}$. Un des correspondants d'Albert Dauzat, le sergent Félix Bertrand, témoigna de la dissémination du français-tirailleur qui était enseigné aux combattants sénégalais afin de les doter des premiers rudiments de l'instruction militaire. Dans les régiments de tirailleurs sénégalais, en effet, la question de la compréhension des ordres donnés par des cadres, officiers et sous-officiers, en majeure partie français, nécessita la recherche de solutions adaptées à la complexité de la situation de polyphonie langagière ${ }^{17}$. Un français simplifié fut notamment adopté, dont Félix Bertand recueillit la trace en 1917 sur la Côte d'Azur : "De Marseille à Menton (Toulon, Fréjus et Saint-Raphaël) on entend des mots et des formules-clichés, passés dans le civil par le canal des cantiniers et du personnel civil employé dans les hôpitaux sénégalais : il y a bon, il n'y a pas bon, il y a moyen, il n'y a pas moyen, faire avancement (attaquer), il y a gagné caisse (mourir), il y a gagné petite voiture (même sens), il y a gagné petit (accoucher) 18". Quelques jours plus tard, il ajoutait à cette liste deux entrées du registre de la sexualité : "le bengala : la verge, la queue" et "casser coco : coïter 19 ".

14. Lettre d'Antonin Duraffour, agrégé de grammaire, officier interprète à l'EM du CA à Albert Dauzat, le 21 avril 1917, ibid. 15. Lettre de G. Mayer, trésorerie aux armées, secteur 71, à Albert Dauzat, le 20 mai 1917, ibid. 16. Voir Emmanuelle Cronier, Permissionnaires dans la Grande Guerre, Paris, Belin, pp. 62-63. 17. Voir Cécile Van den Avenne, "Bambara et français-tirailleur. Une analyse de la politique linguistique au sein de l'armée coloniale française : la Grande Guerre et après", in Documents pour l'histoire du français langue étrangère ou seconde, ${ }^{\circ} 35$, décembre 2005, pp. 123-150. 18. Archives de la BDIC déjà citées, lettre du sergent Bertrand à Albert Dauzat, Menton, le 21 juin 1917. 19. Ibid. 


\section{Une persistance \\ des stéréotypes racistes}

L'intégration de ce vocabulaire d'origine coloniale, dont les linguistes ont montré la dissémination dans le français oral populaire, s'est révélée profondément ambiguë. Pour Sainéan, Dauzat ou Esnault, tenons-nous-en à ces trois exemples, il s'agissait d'unir dans l'effort de guerre l'ensemble des combattants, quelle que fût leur origine, et de rendre hommage au courage et au dévouement des troupes coloniales. Cette intention était clairement perceptible dans le commentaire de Lazare Sainéan, qui attribua en 1915 aux soldats coloniaux le substantif hautement valorisant, car synonyme de courage et de virilité, de "poilus". Ainsi la lexicographie du temps de guerre semble apporter sa contribution au vaste discours qui insistait alors sur la participation des colonies à l'œuvre commune de lutte contre la barbarie. Une barbarie incarnée du côté français dans le mot "Boche" (Allemand), un terme qui fit couler beaucoup d'encre chez les spécialistes de I'histoire de la langue ${ }^{20}$. La ligne de partage entre la civilisation et le monde des forces obscures sembla alors se déplacer quelque peu, vu la censure, dans les principaux lexiques de l'argot de la guerre, de termes particulièrement dévalorisants, notamment à l'égard de l'homme noir, couramment traité de “nègre" jusqu'en 1914, et l'absence d'allusions, courantes dans la presse populaire de ce temps, à l'animalité de ce dernier trahie par son goût pour l'anthropophagie. II ne faudrait toutefois pas conclure à partir de ces quelques constats, nécessairement hâtifs, au recul des préjugés dominants sur l'infériorité raciale et intellectuelle des colonisés. L'enquête de Dauzat, qui reste la mieux documentée pour l'historien, fut le produit d'une autocensure qui agit à un double niveau. Les correspondants dissimulèrent soigneusement dans leurs réponses tout indice trop visible de l'ampleur des poncifs à l'égard des colonisés, et lorsqu'ils signalèrent la valeur péjorative de certains mots, cela disparut dans le texte final de l'enquête. Ainsi la remarque du brigadier Marcel Divernesse du $4^{e}$ chasseurs d'Afrique qui signalait que bicot, utilisé pour désigner un Arabe ou même un homme originaire de Tunisie ou d'Algérie, était un terme de mépris ${ }^{21}$, a été gommée du manuscrit de Dauzat. Gaston Esnault, dont l'étude se veut moins épurée, révélait a contrario la profondeur des préjugés racistes véhiculés par certains de ces mots. À l'entrée "bougnoul" par exemple, il précisa que ce mot utilisé dans les régiments brestois dès les années 1890 signifiait un "troupier en tant que corvéable". Issu du mot wolof (Sénégal) bou-gnoul (le Noir), il renvoyait à l'infériorité intellectuelle du Noir, qui de ce fait était facilement exploitable ${ }^{22}$. Dans le même registre, le mot "sidi" appela de la part du linguiste cette définition à double niveau : le sidi est un soldat indigène algérien, et le mot, usuel pendant la Grande Guerre, vient du vocatif arabe sidi, qui signifie "monsieur" avec un sens péjoratif. Et d'ajouter : les "hommes de couleur, êtres de soumission, ont souvent à dire sidi23".

Par conséquent, si la Grande Guerre a constitué un moment propice à l'interculturalité, si les mots empruntés aux langues des colonisés ont acquis pendant le conflit un surcroît de visibilité, et s'ils ont pénétré, sans doute à un moindre degré que l'argot militaire ou l'argot parisien, les usages oraux populaires, ce rapprochement n'a nullement signifié un recul des préjugés racistes. Bien au contraire, la familiarité nouvelle avec un vocabulaire colonial a peut-être enraciné un peu plus la conviction, déjà largement répandue avant 1914, de l'infériorité ontologique des colonisés. 